\title{
Goniurosaurus chengzheng sp. nov., a new species of Leopard Gecko from Guangxi, China (Squamata: Eublepharidae)
}

\author{
XIAO-YU ZHU ${ }^{1}$, YI-JIAO LIU ${ }^{2,5}$, YUN BAI ${ }^{2,6}$, CRISTIAN ROMÁN-PALACIOS ${ }^{3}$, \\ ZHENG LI' ${ }^{4 *} \&$ ZHU-QING HE ${ }^{1 *}$ \\ ${ }^{1}$ Museum of Biology, School of Life Sciences, East China Normal University, Shanghai 200241, China. \\ "E"zqhe@bio.ecnu.edu.cn; ๑ https://orcid.org/0000-0003-4304-767X \\ ${ }^{2}$ School of Life Sciences, East China Normal University, Shanghai 200241, China. \\ ${ }^{3}$ Department of Ecology and Evolutionary Biology, University of Arizona, Tucson 85719, USA. \\ (1) https://orcid.org/0000-0003-1696-4886 \\ ${ }^{4}$ Department of Integrative Biology, University of Texas at Austin 78705, USA.

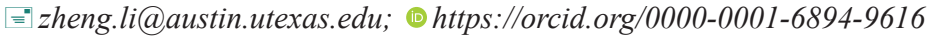 \\ $5 \odot$ https://orcid.org/0000-0002-2169-5628 \\ ${ }^{6} \odot$ https://orcid.org/0000-0003-4849-8429 \\ ${ }^{*}$ Corresponding author
}

\begin{abstract}
Six species of geckos in the genus Goniurosaurus have been recorded from Guangxi, China. Here we describe a new species, Goniurosaurus chengzheng sp. nov. The new species is similar to allied species from Guangxi, but unique in a combination of the following characters: (1) four body bands with three between limb insertions; (2) precloacal pores 20; (3) body color reddish- brown; (4) snout to eye distance: eye to ear distance $<1$. We used the mitochondrial genes $16 S$ and $c y t b$ to confirm the distinctiveness of the species and place it within a molecular phylogeny of Goniurosaurus. The type specimens are deposited in the Museum of Biology, East China Normal University (ECNU).
\end{abstract}

Key words: gecko, taxonomy, molecular phylogeny, karst, Guangxi, China

\section{Introduction}

The genus Goniurosaurus currently comprises 23 described species (Mocquard 1897; Orlov \& Darevsky 1999; Grismer et al. 1999, 2002; Orlov et al. 2008; Ziegler et al. 2008; Wang et al. 2010, 2013, 2014; Honda et al. 2014; Yang \& Chan 2015; Honda \& Ota 2017; Zhou et al. 2018; Zhu et al. 2020a, 2020b; Qi et al. 2020a, 2020b). Recent molecular phylogenetic analyses have indicated the existence of four major lineages of Goniurosaurus (Liang et al. 2018; Zhu et al. 2020a; Qi et al. 2020b), namely the G. kuroiwae, G. lichtenfelderi, G. luii, and G. yingdeensis groups. In 2020, one new species was described in the G. lichtenfelderi group (G. kwanghua, Zhu et al. 2020b), one in the G. luii group (G. gezhi, Zhu et al. 2020a), and two in the G. yingdeensis group (G. gollum and G. varius, Qi et al. 2020a, 2020b). The G. luii group is the most diverse group of Goniurosaurus and its species are primarily found in the karst environment of Guangxi and Guizhou provinces of China and adjacent Northern Vietnam (Zhu et al. 2020a). In 2017 an individual was photographed from a new locality in Guangxi during our herpetological surveys (Fig. 1A). The following year, two specimens of Goniurosaurus were collected from the same locality. Morphological and molecular analyses presented in this study support that these individuals belong to a currently undescribed species within the $G$. luii group. 


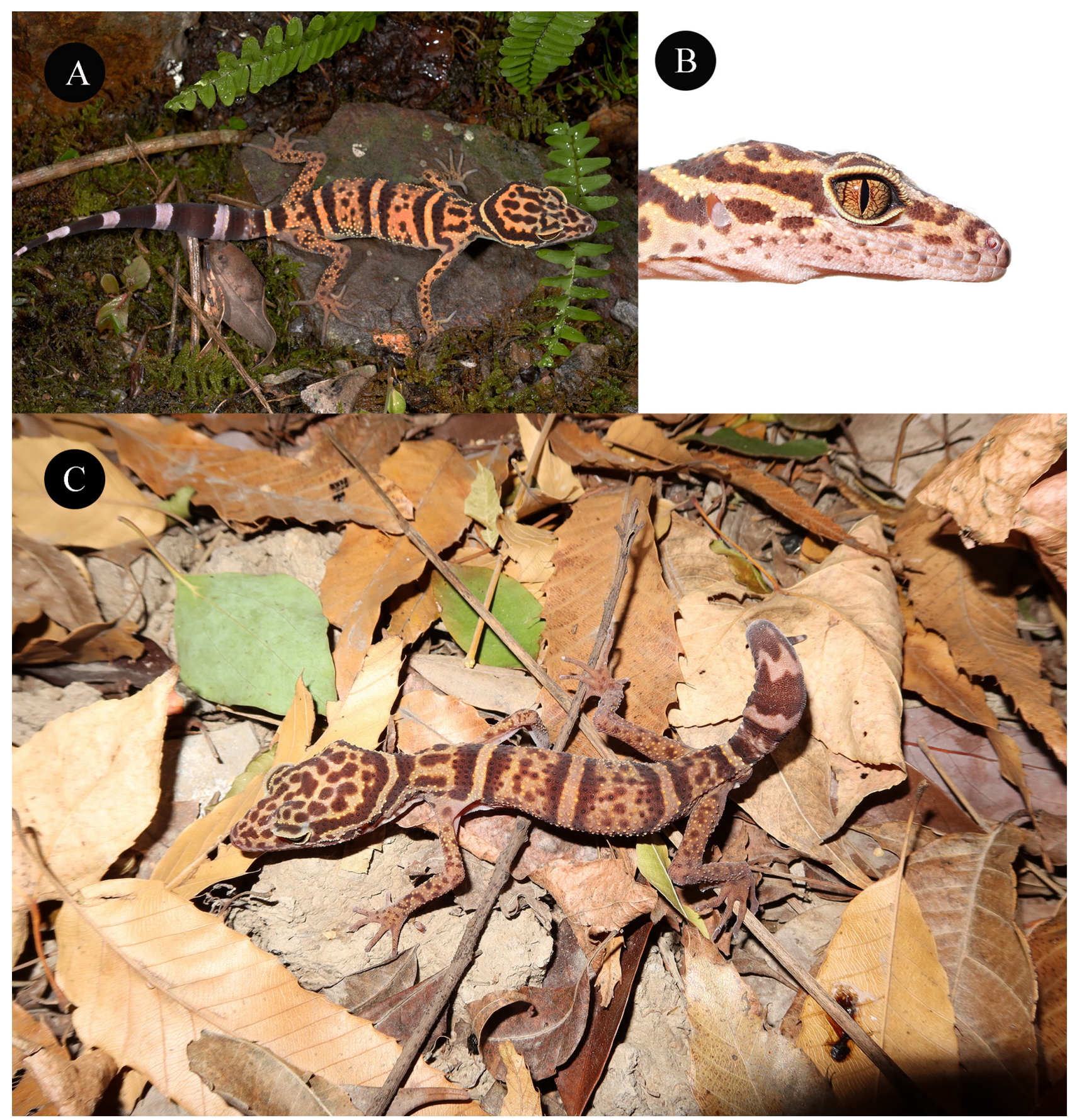

FIGURE 1. Goniurosaurus chengzheng sp. nov. from Guangxi, China. A. Adult female (photographed in 2017, but not collected); B. Scalation and coloration characters of the head of the holotype; C. Adult male, holotype. (photos by Zhu Xiao-Yu)

\section{Methods}

Sampling. We collected specimens of the new species by conducting visual survey encounters in karst areas along the ground at night. Two male individuals of the new species (ECNU-V0068 and ECNU-V0090) were collected. The Personalbio Saliva/Swab Genomic DNA Kit (Shanghai Personalbio Biotechnology Co., Ltd.) was used to collect DNA. We followed the protocol by swabbing the mouth of each individual for about 10 seconds. DNA samples were preserved at room temperature. We preserved the vouchers in $75 \%$ ethanol. Only the general collection locality for each of the examined specimens is provided in Table 1 in order to protect the species from illegal exploitation through the pet trade. All specimens were deposited in the Museum of Biology, East China Normal University (ECNU), which retains full locality data for the specimens. 


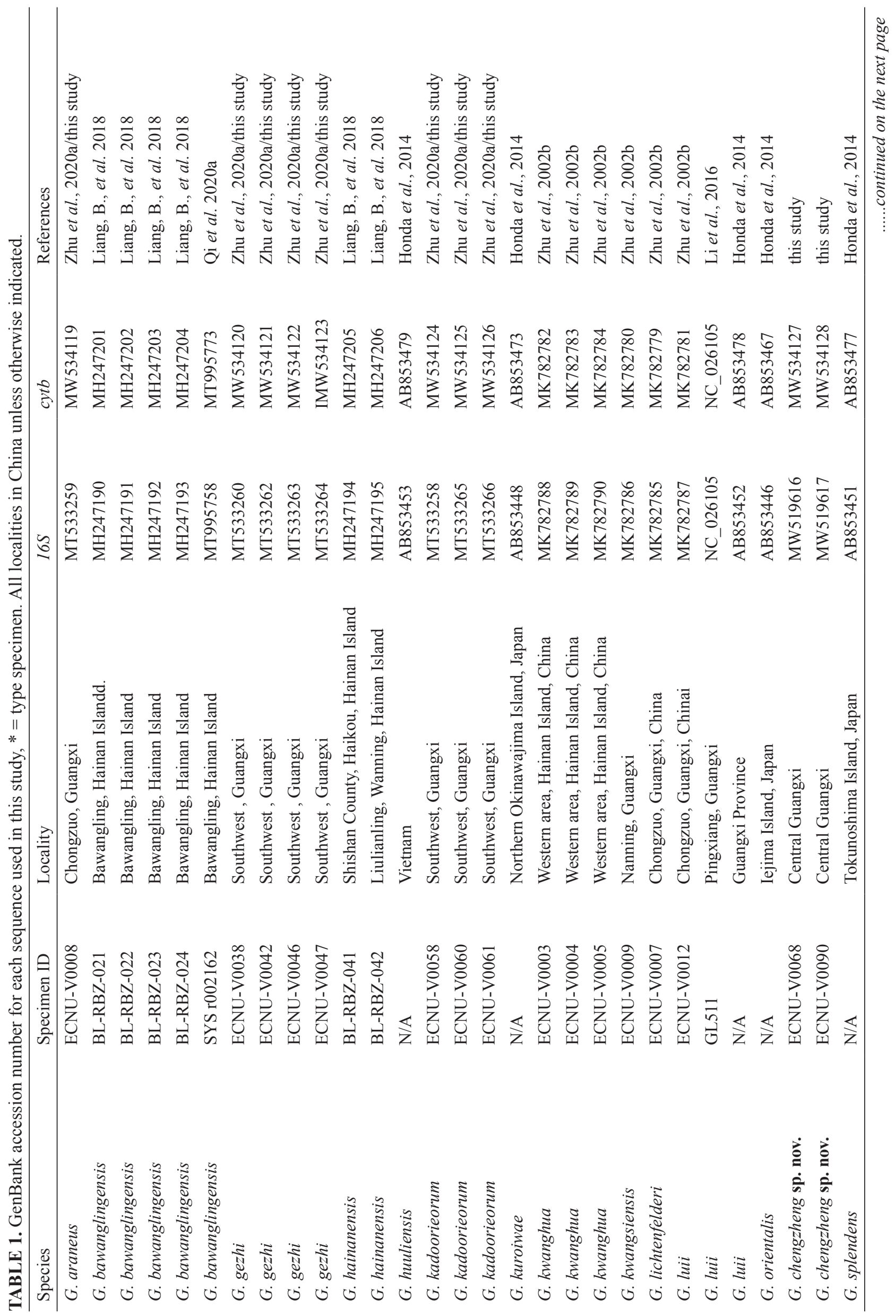




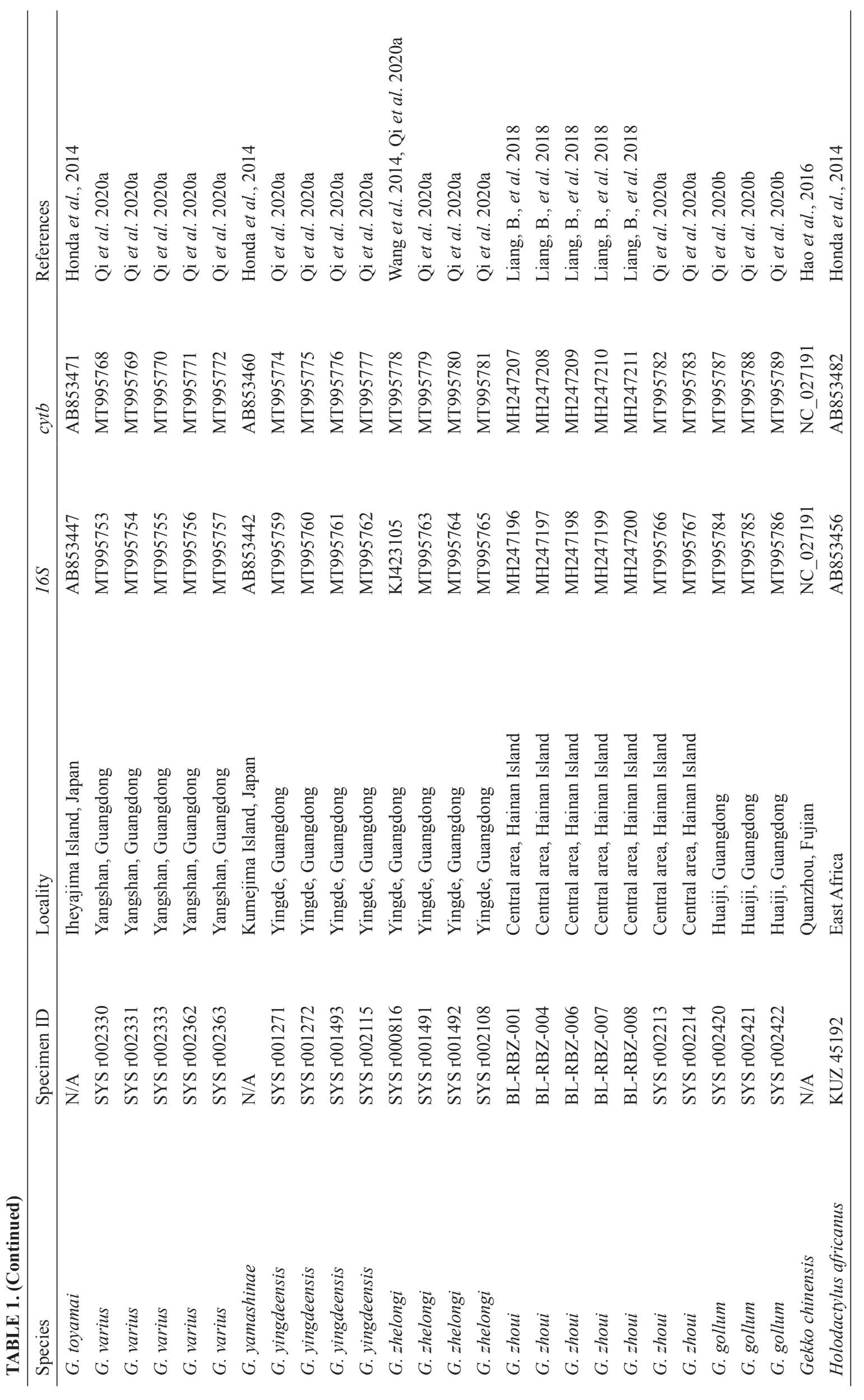


DNA extraction and gene amplification. We sequenced $16 S$ and $c y t b$ mitochondrial gene regions of the new species, three G. kadoorieorum samples, three specimens of G. gezhi, and one G. araneus (Table 1). Total genomic DNA was extracted from the saliva DNA samples according to the manufacturer's instructions. Primers used for PCR amplification of $16 \mathrm{~S}$ are r16S-5L GGTMMYGCCTGCCCAGTG and 16Sbr-H CCGGTCTGAACTCAGATCACGT (Jonniaux \& Kumazawa 2008) and those for $c y t b$ are Cytb-2019F CGAAAAACCAYYGTTGTTATTCAACT and Cytb-2019R AGRTTTGTGATTACGGTTGCGCCT (Zhu et al. 2020b). Amplification conditions for PCR were as follows: pre-denaturation for $2 \mathrm{~min}$ at $94^{\circ} \mathrm{C}, 35$ cycles of $30 \mathrm{~s}$ at $94^{\circ} \mathrm{C}, 30 \mathrm{~s}$ at $55^{\circ} \mathrm{C}$ or $50^{\circ} \mathrm{C}$ for $16 \mathrm{~S}$ and cytb, respectively, $30 \mathrm{~s}$ at $72^{\circ} \mathrm{C}$, and a final extension at $72^{\circ} \mathrm{C}$ for $7 \mathrm{~min}$. All PCR products were sequenced directly with the same primers. GenBank accession numbers are provided in Table 1.

Phylogenetic analyses. Our analyses are mainly based on the published sequence alignment by Zhu et al. (2020b). The sequence alignment analyzed in the current study is equivalent to the one in Zhu et al. (2020b), but included sequences for two specimens of the new species and seven additional specimens of other G. luii group members. Sequence alignment was performed using MEGA version X (Stecher et al. 2020). The resulting alignment included 55 sequences from 21 Goniurosaurus species, two outgroup species (Gekko chinensis Gray and Holodactylus africanus Boettger), and had a total length of $862 \mathrm{bp}$. We inferred the phylogenetic relationships among all 21 Goniurosaurus species in our dataset using stochastic algorithms under maximum likelihood in IQ-TREE (Nguyen et al. 2015) along with a Bayesian approach implemented in MrBayes (Huelsenbeck \& Ronquist, 2001). The bestfitting partitioning scheme across genes was selected using PartitionFinder v2 (Lanfear et al. 2017). Analyses in PartitionFinder were exhaustive (argument "search=all"), assumed branch lengths to be linked across partitions, compared using AIC values, and examined models that were implemented in MrBayes. PartitionFinder identified two independent gene-based partitions in our dataset. Partitioning analyses also assigned a GTR $+\mathrm{G}$ model to $16 \mathrm{~S}$ and GTR $+\mathrm{I}+\mathrm{G}$ to $c y t b$. Maximum-likelihood analyses were conducted in IQ-TREE based on the partitions identified before using PartitionFinder. Support values were estimated based on a total of 1,000 bootstrap pseudoreplicates. Bayesian phylogenies were inferred in MrBayes. For these analyses, we conducted two independent MCMC runs, each with 10 million generations. The maximum clade credibility tree under MrBayes was constructed based on the $90 \%$ of posterior samples (i.e., $10 \%$ burn-in). We confirmed convergence under MrBayes 3.2.4 (Ronquist et al. 2012) in Tracer (Fig. 2). Finally, we estimated uncorrected pairwise $p$-distances between all the samples in our dataset using Mega version X (Stecher et al. 2020). These estimates of genetic distances were used to examine whether the median distances between the new species and all other Goniurosaurus were comparable to those estimates for other species in the dataset. We used the base R package version 4.0.3 (R Core Team 2020) to estimate median $p$ distance and range of values per species based on sequence-specific pairwise distance per species. We repeated the same procedure for $16 S$ and $c y t b$ gene regions.

Morphology. The following measurements were recorded using digital calipers $( \pm 0.01 \mathrm{~mm})$ following Zhou et al. (2018): snout vent length (SVL) from tip of snout to vent; tail length (TaL) from vent to tip of tail; distance between axilla and groin (AG) from posterior edge of forelimb insertion to anterior edge of hindlimb insertion; snout to eye distance (SE) measured from tip of snout to anterior-most point of eye; eye to ear distance (EE) from posterior margin of eye to posterior margin of ear; maximum head width (HW); head length (HL) from tip of snout to posterior margin of ear. Scalation characters recorded are as follows: supralabials (SPL); sublabials/infralabials (SBL); nasal scales surrounding naris (N); internasals (IN); granular scales bordering the internasals (PostIN); postmentals (PM); gular scales bordering the postmentals (GP); eyelid fringe scales or ciliaria (CIL); preorbital scales (PO); granular scales surrounding dorsal tubercles (GST); dorsal longitudinal tubercle rows at midbody (DTR); paravertebral tubercles between limb insertions (TL); scales around midbody (MB); subdigital lamellae under the first finger (LD1) and the fourth finger (LD4); subdigital lamellae under the first toe (LT1) and the fourth toe (LT4); precloacal pores (PP); postcloacal tubercles (PAT). Bilateral scale counts are given as left/right.

\section{Results}

Phylogenetic relationships. Phylogenetic inference under Maximum Likelihood and Bayesian Inference recovered congruent evolutionary relationships among the 21 Goniurosaurus species analyzed in this study (Fig. 1). Overall, our results are in agreement with those in Zhu et al. (2020a), with the major groups in Goniurosaurus being similar to those in previous studies (e.g., Liang et al. 2018). The two samples assigned to G. chengzheng sp. nov. based 


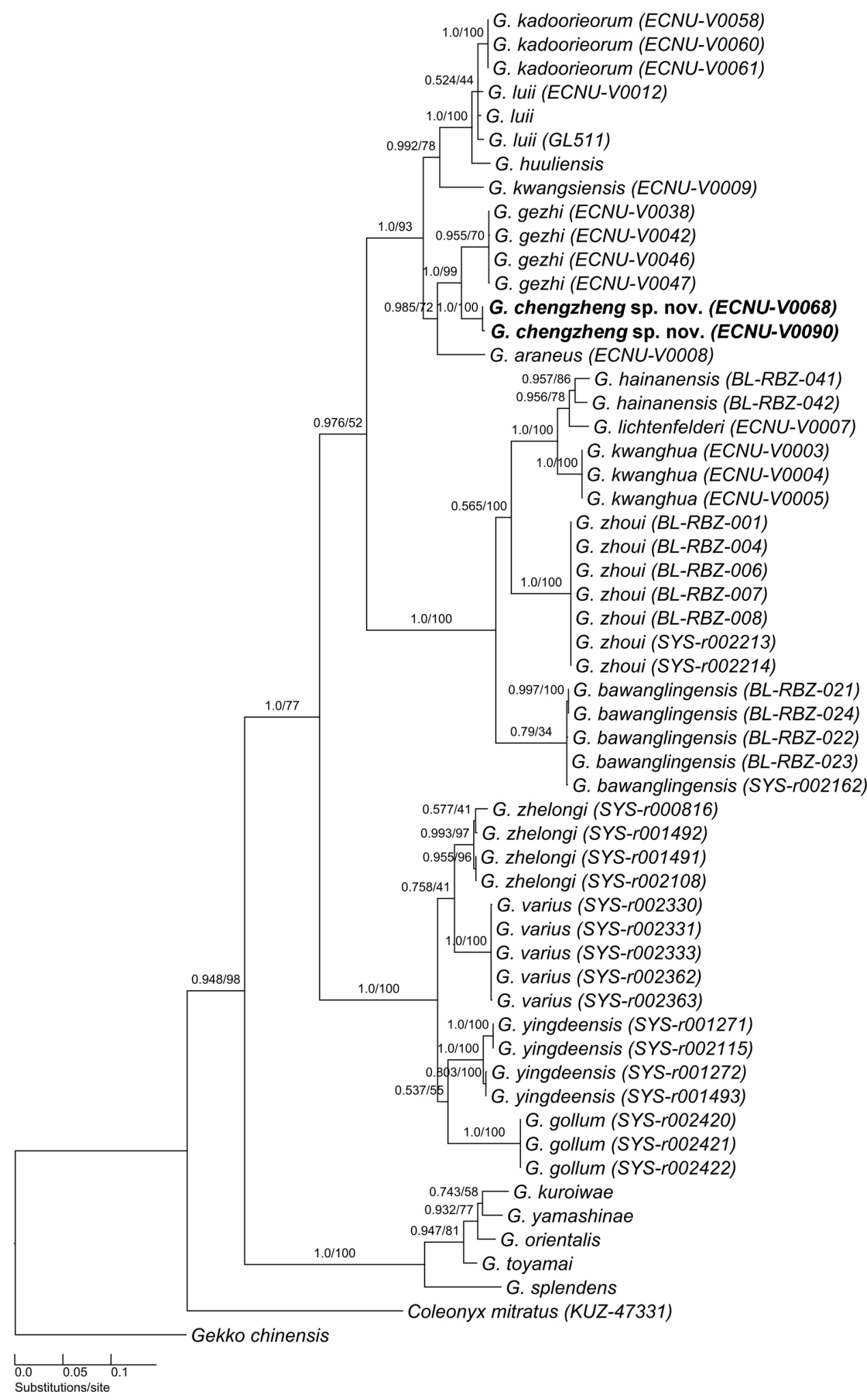

FIGURE 2. Bayesian Inference phylogram showing relationships among 21 Goniurosaurus species and two outgroup taxa. Support values in the form posterior probabilities (Bayesian Inference)/ bootstrap values (maximum likelihood) are shown above branches. The tree is a Bayesian topology. The position of G. chengzheng sp. nov. is highlighted in the tree. 
on morphology formed a strongly supported monophyletic group in our phylogenies (bootstrap $=100 \%$, posterior probability=1). Our phylogenetic analyses suggest that $G$. chengzheng sp. nov. is nested within the $G$. luii clade, with its closest relative being $G$. gezhi (bootstrap $=99 \%$, posterior probability=1; Fig. 2).

We also examined the distribution of genetic distances across Goniurosaurus species based on uncorrected genetic distances for both the $16 S$ and cytb gene regions. Overall, median pairwise genetic distances in G. chengzheng sp. nov. were comparable to those in other species sampled in our dataset (Table 2). Specifically, G. chengzheng sp. nov. has similar values of genetic distances relative to those for G. araneus (16S), G. huuliensis (16S), G. bawanglingensis (cytb), and G. zhelongi (cytb) and even larger than those in G. kwanghua (16S) and G. kwangsiensis ( $16 S$ and $c y t b)$. Thus, estimates of median pairwise genetic distances across species in our dataset suggest that the new species, G. chengzheng sp. nov., genetically differs from other species on a scale that is largely congruent with previously erected species within Goniurosaurus.

TABLE 2. Summary uncorrected $p$-distances for the species of Goniurosaurus analyzed in this study. The median and range (minimum and maximum across pairwise distances across all samples within each species) of genetic distances relative to all other congeners are provided.

\begin{tabular}{lcc}
\hline Species & $\mathbf{1 6 S}$ (median, range) & cytb (median, range) \\
\hline G. araneus & $0.150(0.060-0.221)$ & - \\
G. bawanglingensis & $0.155(0.055-0.223)$ & $0.219(0.158-0.318)$ \\
G. chengzheng sp. nov. & $0.150(0.060-0.232)$ & $0.216(0.098-0.245)$ \\
G. gollum & $0.174(0.057-0.224)$ & $0.242(0.123-0.274)$ \\
G. hainanensis & $0.155(0.023-0.231)$ & $0.237(0.057-0.322)$ \\
G. huuliensis & $0.150(0.013-0.219)$ & $0.235(0.036-0.264)$ \\
G. kadoorieorum & $0.153(0.011-0.215)$ & - \\
G. kuroiwae & $0.207(0.016-0.255)$ & $0.255(0.063-0.284)$ \\
G. kwanghua & $0.148(0.028-0.205)$ & $0.242(0.065-0.284)$ \\
G. kwangsiensis & $0.146(0.046-0.224)$ & $0.213(0.095-0.251)$ \\
G. lichtenfelderi & $0.155(0.023-0.211)$ & $0.238(0.057-0.308)$ \\
G. luii & $0.153(0.011-0.224)$ & $0.229(0.036-0.261)$ \\
G. orientalis & $0.202(0.016-0.235)$ & $0.264(0.063-0.281)$ \\
G. splendens & $0.209(0.063-0.249)$ & $0.294(0.147-0.322)$ \\
G. toyamai & $0.200(0.023-0.240)$ & $0.264(0.063-0.288)$ \\
G. varius & $0.165(0.036-0.211)$ & $0.248(0.076-0.318)$ \\
G. yamashinae & $0.202(0.016-0.237)$ & $0.268(0.063-0.294)$ \\
G. yingdeensis & $0.170(0.048-0.206)$ & $0.242(0.082-0.305)$ \\
G. zhelongi & $0.181(0.036-0.255)$ & $0.219(0.076-0.288)$ \\
G. zhoui & $0.164(0.055-0.207)$ & $0.229(0.144-0.294)$ \\
\hline
\end{tabular}

\section{Systematics}

Goniurosaurus chengzheng Zhu, Li \& He sp. nov.

(Figs. 1, 3)

Holotype. Adult male (ECNU-V0090), from central Guangxi, China, 100-250 m in altitude. Exact locality withheld because of conservation concerns, available to qualified researchers upon request. Collected during July 2018 by Zhu Xiao-Yu.

Paratypes. Subadult male (ECNU-V0068), same data as holotype.

Diagnosis. Goniurosaurus chengzheng sp. nov. differs from other congeners by a combination of the following characters: SE shorter than EE (Table 3); one nuchal loop, four body bands; number of precloacal pores 20; body color reddish brown in life (Table 4). 


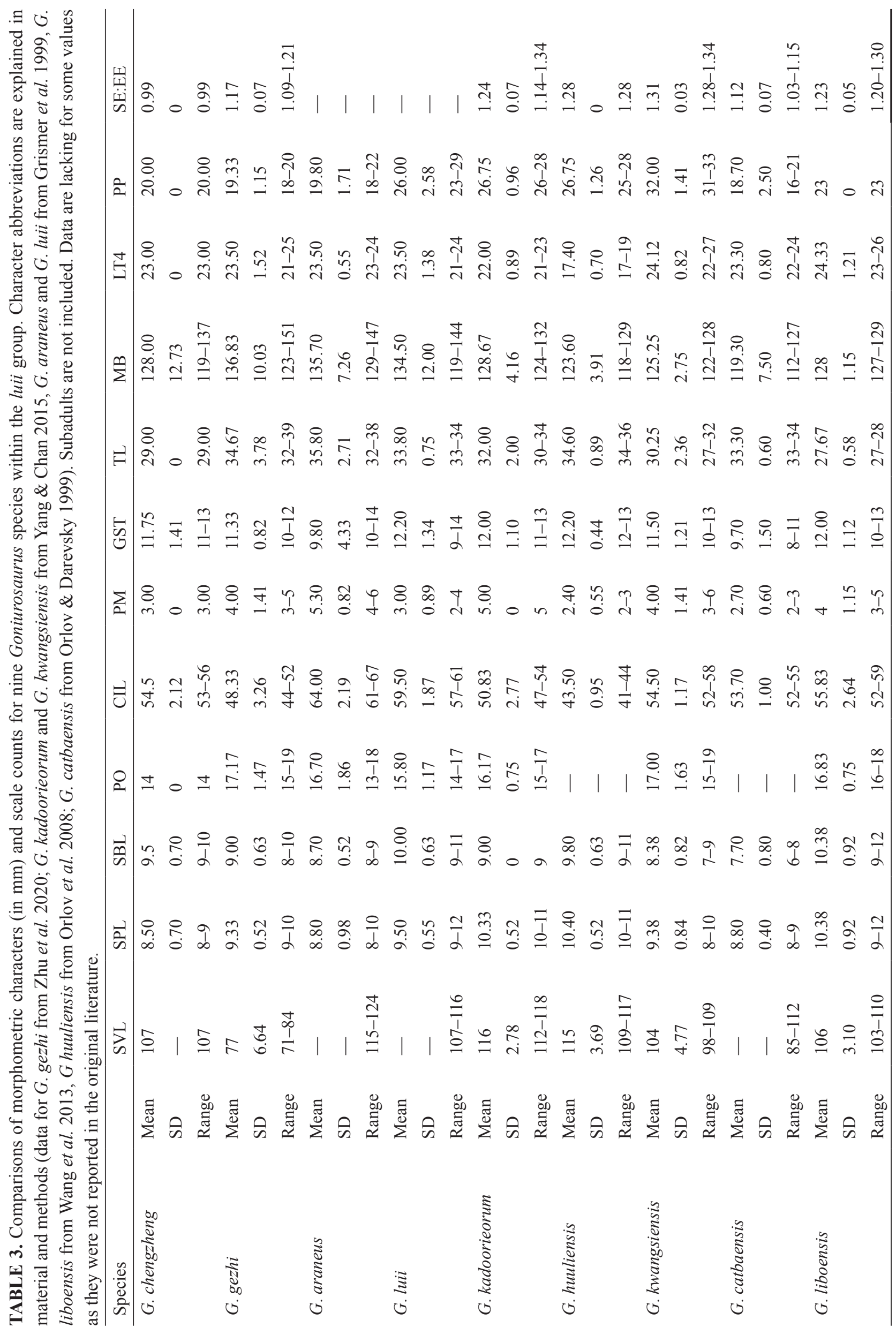


TABLE 4. Diagnostic characters of color pattern distinguishing Goniurosaurus chengzheng from congeners.

\begin{tabular}{llll}
\hline Taxon & dorsal color of body and limbs & color of iris & color of nuchal loops \\
\hline G. chengzheng & reddish brown & yellow & reddish brown \\
G. luii & grey-brown to dull-white & brilliant orange & dull yellow \\
G. araneus & dull yellow-grey & dark brown & yellowish \\
G. huuliensis & brown with dark & red-brown & pink or orange \\
G. liboensis & gray-brown & gray & white \\
G. catbaensis & grey-brown to pale brown & orange-brown & dull yellow \\
G. gezhi & grey & brown & pale yellow \\
$\boldsymbol{G}$. kwangsiensis & yellowish brown & light orange yellow & yellow \\
G. kadoorieorum & greyish lilac & olive green & greyish \\
\hline
\end{tabular}

Description. Holotype. ECNU-V0090, adult male; SVL: 107.08 mm; TaL (regenerated): 62.15 mm; AG: 46.95 mm; SE 11.53 mm; EE: 11.70 mm; HW: 20.57 mm; HL: 29.12 mm; SVL: AG 2.28; SVL: HL: 3.68; HL: HW 1.42; SE: EE: 0.99; head triangular, wider than neck, covered with uniform granular scales interspersed with tubercles on top of head; conspicuous row of enlarged supraorbital tubercles; external nares bordered by $8 / 8$ nasals, anteriorly by prenasal, dorsolaterally by supranasal and two granular scales, dorsally by one internasal, posteriorly by four smaller granular scales; prenasals with long recurved ventral portion; supranasals in contact at the midline; separated by one internasal (Fig. 3B); supralabials 9/8, rectangular; eyes relatively large, pupils vertical; eyelid fringe scales 53/56; a fold of skin originating in suborbital region extends posteroventrally across angle of jaw; external auditory meatus elliptical with long axis directed dorsoventrally; tympanum deeply recessed, bordered anteriorly by two spinose scales; mental triangular, bordered laterally by first infralabial and posteriorly by three postmentals; postmentals collectively bordered by six gular scales (Fig. 3C); infralabials rectangular 10/9. Neck narrower than body, covered with uniform granular scales interspersed with conical tubercles on nape; dorsal body tubercles surrounded by 11-13 smooth granular scales; 21 longitudinal rows of dorsal tubercles at midbody; 29 paravertebral tubercles between limb insertions, distinct vertebral row of scales absent. Body relatively thin, covered with granular scales grading ventrally into larger flattened subimbricate ventral scales; 119-137 scales around midbody; larger ventral scales grade abruptly into smaller granular scales immediately anterior to vent; 20 pore-bearing precloacal scales in a continuous transverse series extending onto proximal regions of thighs (Fig. 3D); one enlarged postcloacal tubercle laterally on each side at level of vent. Limbs covered dorsally with granular scales interspersed with closely spaced tubercles and ventrally with flat, subimbricate scales; hind limbs more robust and longer than forelimbs; deep axillary pockets present; subdigital lamellae wide, 8/8 on first finger, 17/16 on fourth finger, 11/11 on first toe, $23 / 23$ on fourth toe. Tail regenerated and gradually narrowing to the tip.

Coloration. Dorsal ground color of head, body, and limbs reddish brown, bearing irregularly shaped small black blotches, black blotches on head; iris yellow; nuchal loop reddish brown, posterior margin rounded, not pointed; longitudinal black lines between nuchal loop and the first body band; three body bands between limb insertions, one postsacral band on tail base; all bands are reddish brown; ventral surfaces of head, body, and limbs dull white.

Variation. Measurements and scalation data of the type series are provided in Table 5. The paratype (a subadult) largely matches the overall scalation and coloration characters of the holotype, however, it has two internasals and three PostIn.

Comparisons. Goniurosaurus chengzheng sp. nov. is most similar to G. gezhi, but has a proportionally smaller snout (SE:EE; see Table 3), and differs in having reddish-brown body color (versus orange or yellow). It also differs from G. lichtenfelderi by having three body bands (versus four body bands). It differs from G. luii, G. huuliensis, G. kadoorieorum, G. kwangsiensis, and G. liboensis by having 20 precloacal pores as opposed to 23-33 (Table 3). It differs from $G$. araneus by having no blotches on the body (versus black blotches).

Distribution and Life History. Goniurosaurus chengzheng sp. nov. is only known from central Guangxi, China at 100-250 m elevation. The known distributions of G. chengzheng sp. nov. and G. gezhi are not overlapping and are separated by the Zuo River. Specimens of the new species were found on limestone and soil slopes near a swallet at night. Goniurosaurus chengzheng sp. nov. was found to co-occur with Moellendorf's Rat Snake (Elaphe moellendorffi Boettger) and a terrestrial crab (Tiwaripotamon sp.) in the limestone area.

Etymology. The specific epithet chengzheng is from the Chinese phonetic alphabet 诚正, which was noted in 
the ancient work of Chinese literature Daxue around 2000 years ago. It means having true ideas to put one's mind in a proper and well-ordered condition. The word chengzheng is often used in parallel with gezhi. Given this new species is sister to G. gezhi in our molecular phylogeny, we use chengzheng for the specific epithet. For the common name, we suggest “Chengzheng Cave Gecko, 诚正睑虎”.
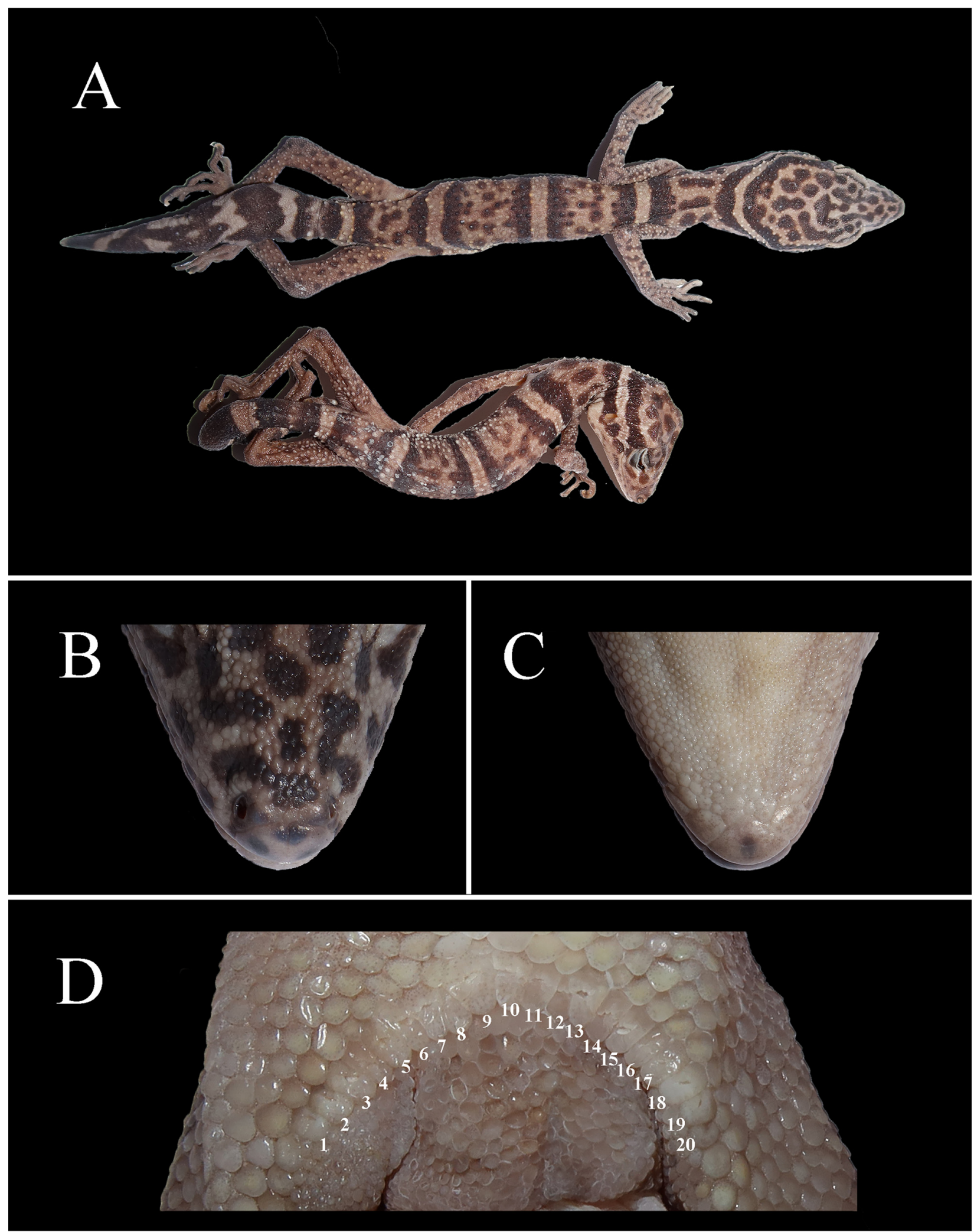

FIGURE 3. Goniurosaurus chengzheng sp. nov. A. Dorsal view of holotype, ECNU-V0090 (left) and paratype, ECNU-V0068 (right); B. Ventral view of the chin, holotype; C. Dorsal view of the snout tip, holotype; D. Precloacal region, with the precloacal pores numbered. 
TABLE 5. Morphological measurements (in $\mathrm{mm}$ ) and scalation characters of the type specimens of Goniurosaurus chengzheng sp. nov. Abbreviations are defined in the Materials and Methods. * = regenerated tail.

\begin{tabular}{|c|c|c|}
\hline & HOLOTYPE & PARATYPE \\
\hline & ECNU-V0090 & ECNU-V0068 \\
\hline sex & adult male & subadult male \\
\hline SVL & 107.08 & 77.35 \\
\hline $\mathrm{TaL}$ & $62.15^{*}$ & $47.82 *$ \\
\hline $\mathrm{AG}$ & 46.95 & 40.50 \\
\hline HL & 29.12 & 26.57 \\
\hline HW & 20.57 & 19.85 \\
\hline SE & 11.53 & 11.08 \\
\hline $\mathrm{EE}$ & 11.70 & 11.69 \\
\hline SVL:HL & 3.68 & 2.91 \\
\hline SVL:AG & 2.28 & 1.91 \\
\hline HL:HW & 1.42 & 1.34 \\
\hline SE:EE & 0.99 & 0.95 \\
\hline SPL & $9 / 8$ & $10 / 10$ \\
\hline SBL & $10 / 9$ & $8 / 10$ \\
\hline $\mathrm{N}$ & $8 / 8$ & $8 / 9$ \\
\hline IN & 1 & 2 \\
\hline PostIN & 2 & 3 \\
\hline PM & 3 & 3 \\
\hline GP & 6 & 7 \\
\hline PO & $14 / 14$ & $18 / 17$ \\
\hline CIL & $53 / 56$ & $54 / 59$ \\
\hline MB & 119-137 & 133-141 \\
\hline GST & $11-13$ & $11-12$ \\
\hline TL & 29 & 29 \\
\hline DTR & 21 & 19 \\
\hline LD1 & $8 / 8$ & $8 / 8$ \\
\hline LD4 & $17 / 16$ & $20 / 21$ \\
\hline LT1 & $11 / 11$ & $12 / 9$ \\
\hline LT4 & $23 / 23$ & $22 / 24$ \\
\hline PP & 21 & - \\
\hline PAT & 1 & 1 \\
\hline
\end{tabular}

\section{Discussion}

In this study, we added $16 S$ and cytb sequence data for four species of Goniurosaurus (G. chengzheng sp. nov., G. gezhi, G. kadoorieorum, G. araneus), and presented an updated phylogeny for $91 \%$ of the known Goniurosaurus species including new species that were published in 2020 (Zhu et al. 2020b). The topology is similar to that inferred in previous studies (e.g., Liang et al. 2018).

Our molecular analyses suggest $G$. chengzheng sp. nov. belongs to the G. luii group and is distinct from other congeners. Goniurosaurus chengzheng sp. nov. is morphologically most similar to G. gezhi and our molecular analyses also retrieve these two taxa as sister species. Genetic distances between $G$. chengzheng sp. nov. and congeners are comparable to those for other species within the same genus (Table 2), and also similar to uncorrected distances between species in other gekkotan genera (Bauer and Lamb 2002; Gamble et al. 2012; Metallinou et al. 2012). The 
geographical ranges of G. gezhi and G. chengzheng sp. nov. are separated by the Zuo River, which may serve as a barrier to gene flow between these two species.

This new species is only found in karst habitat in Guangxi province of China. Together with four Goniurosaurus species recently discovered in China (Zhu et al. 2020a, 2020b; Qi et al. 2020a, 2020b), this suggests potential unknown Goniurosaurus species remain to be discovered in the karst landscapes of China and northern Vietnam. This karst area is known for the high degree of endemism (Luo et al. 2016; Wang et al. 2017). The discovery of $G$. chengzheng sp. nov. and other species that have recently been found in the limestone area in Guangxi (e.g., Xu et al. 2021, Meng et al. 2020) highlights that the conservation efforts need to be improved to protect many endemic species living in this region. Goniurosaurus is known to be illegally collected for pet trade (Grismer et al. 1999; Stuart et al. 2006; Lindenmayer \& Scheele 2017). To protect this new species, we follow the current community standard to withhold detailed type locality information (Yang \& Chan 2015; Zhu et al. 2020a, 2020b).

\section{Acknowledgments}

We thank Aaron M. Bauer, L. Lee Grismer, Chris R. Reardon, and an anonymous reviewer for their helpful comments and suggestions on the manuscript. We thank Yuanjun Li for his idea for the common name.

\section{References}

Bauer, A.M. \& Lamb, T. (2002) Phylogenetic relationships among members of the Pachydactylus capensis group of southern African geckos. African Zoology, 37 (2), 209-220. https://doi.org/10.1080/21564574.2005.9635525

Gamble, T., Colli, G.R., Rodrigues, M.T., Werneck, F.P. \& Simons, A.M. (2012) Phylogeny and cryptic diversity in geckos (Phyllopezus; Phyllodactylidae; Gekkota) from South America's open biomes. Molecular Phylogenetics and Evolution, 62 (3), 943-953. https://doi.org/10.1016/j.ympev.2011.11.033

Grismer, L.L., Haitao, S., Orlov, N.L. \& Ananjeva, N.B. (2002) A new species of Goniurosaurus (Squamata: Eublepharidae) from Hainan Island, China. Journal of Herpetology, 36 (2), 217-225. https://doi.org/10.1670/0022-1511

Grismer, L.L., Viets, B.E. \& Boyle, L.J. (1999) Two new continental species of Goniurosaurus (Squamata: Eublepharidae) with a phylogeny and evolutionary classification of the genus. Journal of Herpetology, 33 (3), 382-393. https://doi.org/10.2307/1565635

Hao, S., Ping, J. \& Zhang, Y. (2016) Complete mitochondrial genome of Gekko chinensis (Squamata, Gekkonidae). Mitochondrial DNA Part A, 27 (6), 4226-4227. https://doi.org/10.3109/19401736.2015.1022751

Honda, M. \& Ota, H. (2017) On the live coloration and partial mitochondrial DNA sequences in the topotypic population of Goniurosaurus kuroiwae orientalis (Squamata: Eublepharidae), with description of a new subspecies from Tokashikijima Island, Ryukyu Archipelago, Japan. Asian Herpetological Research, 8 (2), 96-107. https://doi.org/10.16373/j.cnki.ahr.170003

Honda, M., Kurita, T., Toda, M. \& Ota, H. (2014) Phylogenetic relationships, genetic divergence, historical biogeography and conservation of an endangered gecko, Goniurosaurus kuroiwae (Squamata: Eublepharidae), from the Central Ryukyus, Japan. Zoological Science, 31, 309-320. https://doi.org/10.2108/zs130201

Huelsenbeck, J.P. \& Ronquist, F. (2001) MRBAYES: Bayesian inference of phylogenetic trees. Bioinformatics, 17 (8), $754-$ 755. https://doi.org/10.1093/bioinformatics/17.8.754

Lanfear, R., Frandsen, P.B., Wright, A.M., Senfeld, T. \& Calcott, B. (2017) PartitionFinder 2: new methods for selecting partitioned models of evolution for molecular and morphological phylogenetic analyses. Molecular Biology and Evolution, 34 (3), 772-773. https://doi.org/10.1093/molbev/msw260

Li, H.-M., Hou, L.-X., Zhang, Y., Guo, D.-N., Liu, Y.-J. \& Qin, X.-M. (2016) Complete mitochondrial genome of Goniurosaurus luii (Squamata, Eublepharidae). Mitochondrial DNA Part A, 27 (3), 2131-2132. https://doi.org/10.3109/19401736.2014.982591

Liang, B., Zhou, R.-B., Liu, Y.-L., Chen, B., Grismer, L.L. \& Wang, N. (2018) Renewed classification within Goniurosaurus (Squamata: Eublepharidae) uncovers the dual roles of a continental island (Hainan) in species evolution. Molecular Phylogenetics and Evolution, 127, 646-654. 
https://doi.org/10.1016/j.ympev.2018.06.011

Lindenmayer, D. \& Scheele, B. (2017) Do not publish. Science, 356 (6340), 800-801.

https://doi.org/10.1126/science.aan 1362

Luo, Z.H., Tang, S.H., Jiang, Z.G., Chen, J. Fang, H.H. \& Li, C.W. (2016) Conservation of terrestrial vertebrates in a global hotspot of karst area in southwestern China. Scientific Reports, 6, 25717.

https://doi.org/10.1038/srep25717

Meng, T., Nong, D.-X., Yuan, Q. \& Liu, Y. (2020) Daphniphyllum peltatum, a new species of Daphniphyllaceae from limestone areas in southwestern Guangxi, China. Taiwania, 265 (2), 232-236. https://doi.org/10.6165/tai.2020.65.232

Metallinou, M., Arnold, E.N., Crochet, P.A., Geniez, P., Brito, J.C., Lymberakis, P., Baha, E.D.S., Sindaco, R., Robinson, M. \&Carranza, S. (2012) Conquering the Sahara and Arabian deserts: systematics and biogeography of Stenodactylus geckos (Reptilia: Gekkonidae). BMC Evolutionary Biology, 12 (1), 258. https://doi.org/10.1186/1471-2148-12-258

Minh, B.Q., Schmidt, H.A., Chernomor, O., Schrempf, D., Woodhams, M.D., Von Haeseler, A. \& Lanfear, R. (2020) IQ-TREE 2: New models and efficient methods for phylogenetic inference in the genomic era. Molecular Biology and Evolution, 37 (5), 1530-1534. https://doi.org/10.1093/molbev/msaa015

Mocquard, F. (1897) Notes herpétologiques. Bulletin du Museum National d'Histoire Naturelle, 3 (6), $211-217$. https://doi.org/10.5962/bhl.part.19256

Nguyen, L.T., Schmidt, H.A., Von Haeseler, A. \& Minh, B.Q. (2015) IQ-TREE: a fast and effective stochastic algorithm for estimating maximum-likelihood phylogenies. Molecular Biology and Evolution, 32 (1), 268-274. https://doi.org/10.1093/molbev/msu300

Orlov, N.L. \& Darevsky, I.S. (1999) Description of a new mainland species of Goniurosaurus genus, from the north-eastern Vietnam. Russian Journal of Herpetology, 6, 72-78.

Orlov, N.L., Ryabov, S.A., Nguyen, T.T., Nguyen, Q.T. \& Ho, T.C. (2008) A new species of Goniurosaurus (Sauria: Gekkota: Eublepharidae) from north Vietnam. Russian Journal of Herpetology, 15 (3), 229-244.

Qi, S., Grismer, L. L., Lyu Z.-T., Zhang, L., Li, P. P. \& Wang, Y.-Y. (2020a) A definition of the Goniurosaurus yingdeensis group (Squamata: Eublepharidae) with the description of a new species. Zookeys, 986, 127-155. https://doi.org/10.3897/zookeys.986.47989

Qi, S., Wang, J., Grismer, L.L., Chen, H.-H., Lyu, Z.-T. \& Wang, Y.-Y. (2020b) The Stoor Hobbit of Guangdong: Goniurosaurus gollum sp. nov., a cave-dwelling Leopard Gecko (Squamata, Eublepharidae) from South China. ZooKeys, 991, $137-153$. https://doi.org/10.3897/zookeys.991.54935

R Core Team (2020) R: A language and environment for statistical computing. R Foundation for Statistical Computing, Vienna, Austria. Available from: https://www.R-project.org/ (accessed 14 June 2021)

Stecher, G. Tamura, K. and Kumar, S. (2020) Molecular Evolutionary Genetics Analysis (MEGA) for macOS. Molecular Biology and Evolution, 37 (4), 1237-1239. https://doi.org/10.1093/molbev/msz312

Stuart, B.L., Rhodin, A.G., Grismer, L.L. \& Hansel, T. (2006) Scientific description can imperil species. Science, 312 (5777), 1137. https://doi.org/10.1126/science.312.5777.1137b

Wang, Y.-Y., Jin, M.-J., Li, Y.-L. \& Grismer, L.L. (2014) Description of a new species of Goniurosaurus (Squamata: Eublepharidae) from the Guangdong Province, China, based on molecular and morphological data. Herpetologica, 70, 309-322. https://doi.org/10.1655/HERPETOLOGICA-D-13-00080

Wang, Y.-Y., Yang, J.-H. \& Cui, R.-F. (2010) A new species of Goniurosaurus (Squamata: Eublepharidae) from Yingde, Guangdong Province, China. Herpetologica, 66 (2), 229-240. https://doi.org/10.1655/09-046R2.1

Wang, Y.-Y., Yang, J.-H. \& Grismer, L.L. (2013) A new species of Goniurosaurus (Squamata: Eublepharidae) from Libo, Guizhou Province, China. Herpetologica, 69, 214-226. https://doi.org/10.1655/HERPETOLOGICA-D-12-00084

Xu, W.-B., Yang, P., Huang, Y.-S., Zhang, R. \& Tang, M. (2021) Styrax hwangiae (Styracaceae), a new species endemic to limestone areas of Guangxi, China. Phytotaxa, 478 (1), 171-178. https://doi.org/10.11646/phytotaxa.478.1.13

Yang, J.H. \& Chan, B.P.L. (2015) Two new species of the genus Goniurosaurus (Squamata: Sauria: Eublepharidae) from southern China. Zootaxa, 3980 (1), 67-80. https://doi.org/10.11646/zootaxa.3980.1.4

Zhou, R.-B., Wang, N., Chen, B. \& Liang, B. (2018) Morphological evidence uncovers a new species of Goniurosaurus (Squamata: Eublepharidae) from the Hainan Island, China. Zootaxa, 4369 (2), 281-291. https://doi.org/10.11646/zootaxa.4369.2.8

Zhu, X.Y., Chen, G.Y., Román-Palacios, C., Li, Z. \& He, Z.Q. (2020a) Goniurosaurus gezhi sp. nov., a new gecko species from Guangxi, China (Squamata: Eublepharidae). Zootaxa, 4852 (2), 211-222. https://doi.org/10.11646/zootaxa.4852.2.6 
Zhu, X.-Y., Shen, C.-Z., Liu, Y.-F., Chen, L., Li, Z. \& He, Z.-Q. (2020b) A new species of Goniurosaurus from Hainan Island, China based on molecular and morphological data (Squamata: Sauria: Eublepharidae). Zootaxa, 4772 (2), 349-360. https://doi.org/10.11646/zootaxa.4772.2.6

Ziegler, T., Truong, N.Q., Schmitz, A., Stenke, R. \& Rösler, H. (2008) A new species of Goniurosaurus from Cat Ba Island, Hai Phong, northern Vietnam (Squamata: Eublepharidae). Zootaxa, 1771 (1), 16-30.

https://doi.org/10.11646/zootaxa.1771.1.2 